EPiC Series in Built Environment
Volume 1, 2020, Pages 205-213
Associated Schools of Construction Proceed-
ings of the 56th Annual International Conference

\title{
Accuracy of Drone Image Based Volumetric Surveys
}

\author{
Denise Adjidjonu and Joseph Burgett, PhD. \\ Clemson University \\ Clemson, South Carolina
}

\begin{abstract}
The use of Unmanned Aerial Vehicles (UAVs) in stockpile calculations is becoming more common due to the time savings associated with its use. In this paper, the accuracy of drone-based volumetric surveys using nadir and oblique images were compared with traditional survey methods. Two cardboard pyramids, measuring 14 cubic yards and 5 cubic yards respectively, were used to simulate a stockpile. The pyramids were surveyed with waypoint-assisted flights at $40 \mathrm{ft}$, $125 \mathrm{ft}$, and $350 \mathrm{ft}$ altitudes. Additional oblique images were captured at both sides of the targets and field to supplement the nadir flights. The volumes of the resulting models were then computed with ContextCapture software. This study found that adding oblique images to the nadir surveys did not significantly increase the accuracy of the model volumes. Oblique images on their own also yielded similar results to the nadir images. The accuracy of the models at $125 \mathrm{ft}$ ranged from $0.35 \%$ to $3.03 \%$ from the measured values, while the oblique images were off by $0.44 \%$ to $8.23 \%$ of the actual values.
\end{abstract}

Key Words: Drone, Survey, Stockpile, UAV

\section{Introduction}

The use of drones was exclusive to the military until the 1990s when they became available for commercial use (Vincenzi et al., 2015). The proliferation of consumer grade unmanned aerial vehicles (UAVs) was led primarily by the manufacturers DJI and Parrot, who developed the Phantom and Bebop model drones (Bentley, 2018; DJI, 2019; UAV Coach, 2019). Special apps which can function on multiple mobile devices have also been developed to aid in conducting automated flights, data logging and flight planning. (Vincenzi et al., 2015). In August 2016, the FAA introduced Part 107 of the Federal Aviation Regulations detailing the operational and certification requirements for commercial drone use within the United States(FAA, 2016). Part 107 significantly increased access to drones for commercial application and eliminated the need for acquiring waivers for most commercial and educational purposes. A March 2018 survey by the American Association of Highway and Transportation found that 35 out of 44 state DOTs are deploying UAS for various purposes, including mapping and surveying. Twenty-three DOTs have drone policies and 27 DOTs have full time drone pilots. According to this report, drone use is purported to save time, money, and results in safer, faster and better data collection when compared to traditional methods (AASHTO, 2018). 
UAVs are now being used for a variety of purposes, including land surveys. One emerging technique for earthwork mapping and volumetric surveys is to apply softcopy photogrammetry to imagery acquired by small UAVs. These UAVs are a type of aerial platform that can be used to acquire images, but because of their autonomous capabilities and integrated inertial and imaging systems, are effective for developing high-resolution digital terrain models (DTMs) and orthoimages (Hugenholtz et al., 2014).

\section{Literature Review}

While UAVs have been used extensively for military applications, several civil applications are currently being developed for remote sensing. Recent civil applications include color and infrared images used to identify people rescue operations. In agriculture, UAVs with Near Infrared (NIR) have been used to identify crops from other objects (Ellenberg et al., 2016).

In recent years, digital terrain modelling using photogrammetry based images from UAVs has gained popularity, as it is now possible to improve stability and to allow longer flight time. This approach allows for the fast and easy acquisition of images over a large area, including for terrain with severe variations (Kim et al., 2019). According to Hugenholtz et al.(2014), conventional survey techniques for volume measurement in earth-work projects typically include one of the following: total station, global positioning system (GPS), terrestrial laser scanning (TLS), or airborne light and direction ranging (LIDAR).

Structure-from-motion (SfM) software takes overlapping images and stiches them together to create a 3D model which are used for a wide range of functions. (Aguera-Vega et al., 2016; El Meouche et al., 2016). Past studies have indicated that the aerial stockpile volumetric survey accuracy is close in value to that of LIDAR and GPS/GNSS. Hugenholtz et al., (2014) conducted a case study of a gravel stockpile measuring $252 \mathrm{ft}$ by $150 \mathrm{ft}$ by $22 \mathrm{ft}(77 \mathrm{~m}$ by $46 \mathrm{~m}$ by $7 \mathrm{~m}$ ), using an Aeryon Scout UAV at $330 \mathrm{ft}(100 \mathrm{~m})$ above ground level. The stockpile was surveyed both before and after a portion was excavated. A total of 10 ground control points (GCPs) were used as targets for post-processing which produced results comparable to both TLS and LIDAR data. The percent difference in values from the UAV method to the GPS generated values was $2.6 \%$ pre-excavation and $3.9 \%$ post-excavation (Hugenholtz et al., 2014). Draeyer \& Strecha (2014), also conducted an experiment with Pix4D to determine the level of accuracy of aerial stockpile calculations within multiple different terrain. Their experiment found that the aerial surveying method yielded geo-referenced, highly-detailed orthomosaics, with a mean deviation of $3 \mathrm{~cm}$ (1.18in) when compared to LIDAR surveys.

Kim et al., (2019) also compared the accuracy of nadir and oblique images of a DTM from a UAV using two different algorithms. The researchers collected 100 images of a stockpile using a DJI Matrice 100 with a Zenmuse Z3 camera. The images were captured at $90 \%$ overlap at angles of $-75^{\circ}$, $60^{\circ},-45^{\circ}$, and $-30^{\circ}$ over the targeted area. After generating the DTMs, the combined nadir and oblique images proved to be more detailed than just nadir images. Terrain obstructions were more visible with the combined nadir and oblique images especially at areas of severe drop-offs and/or inclines (Kim et al., 2019).

\section{ContextCapture}

For this study, the researchers used ContextCapture by Bentley Systems as their SfM software.

ContextCapture is a high-volume image processing software that generates models and maps to a 
predetermined spatial position. The images required for processing are usually captured via UAVs and then uploaded into the software. Often the images inputted are geotagged and aero triangulated, which refers to the registration of the image block over the locality's coordinate system. This information is used by the software to generate point clouds, DTMs, orthomaps and the like for analysis by the user. ContextCapture has unique features which enables the user to extract information related to the volume, coordinates and level of accuracy of the data points being analyzed. The data can also be manipulated as well as exported to various other platforms such as Google Earth.

ContextCapture works well with the addition of tie points, GCPs and checkpoints (CPs) to improve model accuracy. A tie point corresponds to the pixels in two or more different photographs, where the pixels represent the projection of the same physical point in the scene (Pix4D, 2018b).

ContextCapture can automatically generate many automatic tie points during the aero triangulation process. GCPs are well identifiable landmarks on the ground of known location. They are used both to reference the photogrammetric model with an established local or national coordinate system as well as improve the overall accuracy of the model (Draeyer \& Strecha, 2014). CPs are similar to GCPs except they are not used to create the model. Instead, CPs are used to assess the absolute accuracy of the model. The marks of the checkpoints are used to estimate its 3D position as well as potential errors. The difference between the initial and the computed positions of the checkpoints gives an estimation of the absolute accuracy of the model in the region (Pix4D, 2018a). The accuracy of a model depends on the average ground resolution (AGR) or ground sampling distance (GSD). The resolution, pixel size or GSD is the size of the projected pixel on the ground. It is directly dependent on the UAV sensor's size and height of flight (El Meouche et al., 2016). Aguera-Vega et al., (2016) suggest that at least 15 GCPs be used on a survey site to cut down on field work time.

\section{Methodology}

The goal of this experiment was to analyze the accuracy of UAS based volumetric surveys using SfM software. A structured experiment in an open field with 31 points, geo-located by a professional land survey crew, was conducted to explore this. The field measured approximately 250 feet by 330 feet, which provided ample room for the UAS to maneuver within the airspace. There was some variation in height in the test area, where the southern portion had a difference in elevation of 7 feet higher than the northern portion.

The project was conducted during day time hours using a Phantom 4 Pro drone from DJI. The Phantom 4 Pro is a commercially available, general-purpose drone that works well to capture photogrammetry data. The Phantom 4 Pro is equipped with a 20 -megapixel camera mounted below a 3 -axis gimbal and has approximately 20mins of flight time (DJI, 2018). It is also equipped with an infrared sensor and a downward vision sensor to detect proximity to foreign objects.

A total of 16 GCPs and 15 CPs were distributed across the project area. GCPs were used to locate the models on the earth. The odd-numbered targets were selected to be GCPs while the even-numbered targets served as the CPs. Figure 1 shows the positions of the GCPs and CPs in the field. The CPs were used to assess the absolute accuracy of the 3D models by estimating each points' 3D position as well as potential errors between the actual and computed positions (Pix4D, 2018a). This process is known as bundle adjustment (Aguera-Vega et al., 2016). 


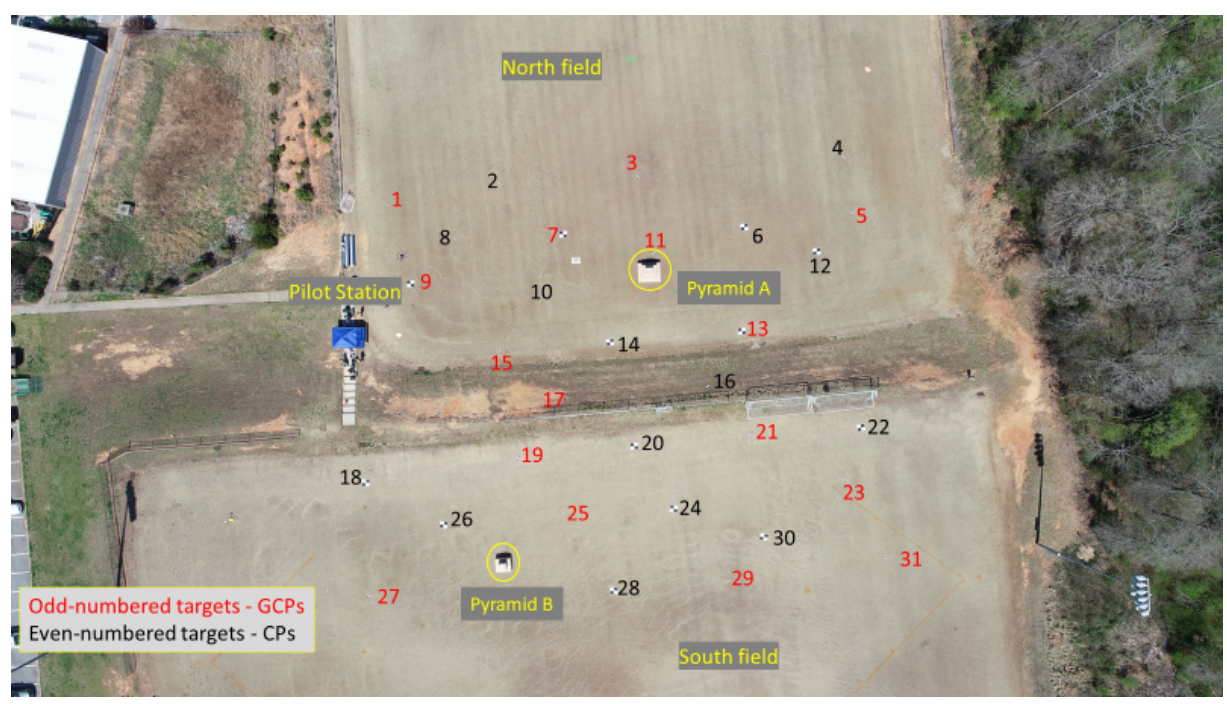

Figure 1. Site setup with the corresponding GCPs and CPs

Two pyramids of varying volumes were used to mimic stockpiles of earth on a construction site (as shown in figure 1). To achieve such volume, several $3 \mathrm{ft}$ by $3 \mathrm{ft}$ by $3 \mathrm{ft}$ (1cu. yd) cardboard boxes were strategically arranged in a pyramid shape. Pyramid A was constructed at the north field using fourteen boxes in total. The final height and volume of Pyramid A measured $9 \mathrm{ft}$ and $14 \mathrm{cu}$. yd, respectively. Pyramid B was constructed at the south field using five boxes in total. The final height and volume of Pyramid B measured $6 \mathrm{ft}$ and 5cu. yd respectively. Figure 2 shows Pyramid A and B for comparison.

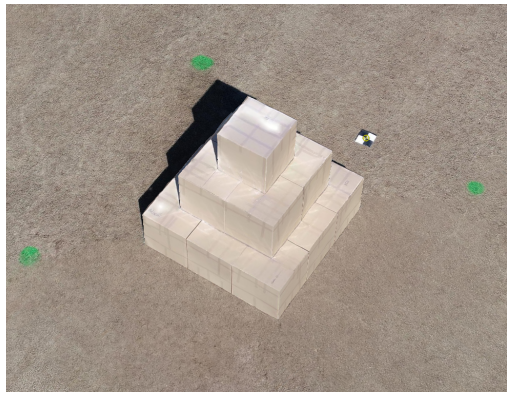

Pyramid A

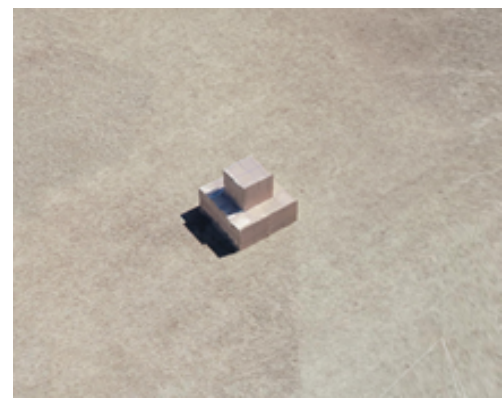

Pyramid B

Figure 2. Pyramid A (14 cu. yd) and Pyramid B (5 cu. yd)

A total of nine waypoint-assisted flights were conducted to capture images on the field for mapping. The drone flights were conducted at $80 \%$ overlap using waypoint-assisted paths. Flights were conducted at 40 feet, 125 feet and 350 feet altitude above the target area. These altitudes were selected due to the limitations from the FAA as well as the software capabilities. As such, 40 feet was the minimum altitude that ContextCapture would compute for the experiment, 350 feet was as high as the FAA would allow with a safety factor, and 125 feet served as the median value between the two altitudes. This sequence was repeated two more times to see how consistent the accuracy of the models was when the flights were repeated, and similar data collected. All images were processed using ContextCapture to generate 3D models for analysis. Table 1 illustrates the sequence of each flight. 
Table 1

Waypoint-assisted nadir flights and their corresponding altitudes

\begin{tabular}{lccc}
\hline Altitude & \multicolumn{3}{c}{ Flight Names } \\
\hline $\mathbf{4 0 f t}$ & $40 \mathrm{~A}$ & $40 \mathrm{~B}$ & $40 \mathrm{C}$ \\
$\mathbf{1 2 5 f t}$ & $125 \mathrm{~A}$ & $125 \mathrm{~B}$ & $125 \mathrm{C}$ \\
$\mathbf{3 5 0 f t}$ & $350 \mathrm{~A}$ & $350 \mathrm{~B}$ & $350 \mathrm{C}$ \\
\hline
\end{tabular}

\section{Data Acquisition}

The Bentley Modeling Drone Capture Guide details the best practices for photo and data acquisition for creating accurate models (Bentley, 2018). According to this guide, transitional photos (obliques) are necessary to aid in the modelling of objects to improve accuracy. Obliques are images captured at a 45-degree angle to the center base of an object and are conducted at a 360-degree orbit around the target. Also, the range of overlap is recommended to be between 50 and $70 \%$ to ensure the robustness of the model. In other words, successive photos must have a minimum angle difference from the object center point at 15 degrees, with a complete orbit comprising at least 24 images captured successfully (Bentley, 2018). In view of these directions, two additional waypoint-assisted flights were conducted at both north and south fields consecutively (Oblique North and Oblique South). These images were later added to the previous nadir images and processed with ContextCapture for analysis.

The volumes of each pyramid were calculated by using the volumetric function on the software. The pyramids were indicated with visible markers to ensure accurate delineation of the critical points for estimation. Figure 3 below provides a sample image of the volume function of ContextCapture, where the green points were selected to facilitate the stockpile computation of each pyramid. The different colored spots on the generated grid demonstrate the cut and fill sections of the stockpile being analyzed.

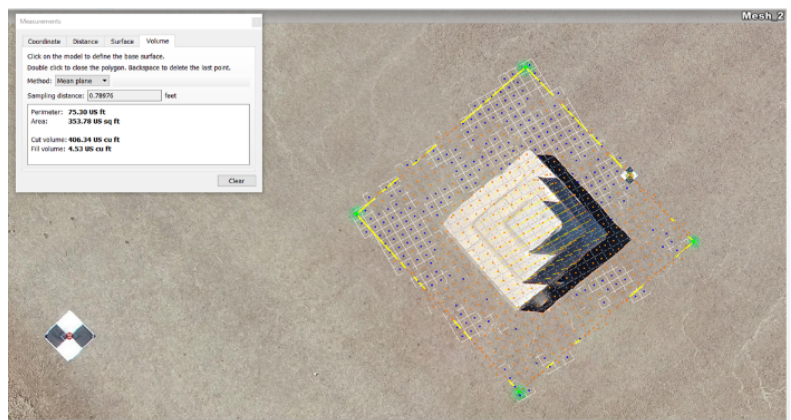

Figure 3. Sample image demonstrating the computed volume of a stockpile

\section{Results \\ Nadir flights only}

In general, all nadir flights at $40 \mathrm{ft}$ failed to yield results. The $40 \mathrm{ft}$ models were distorted and patchy, and hence were unusable by the researchers for analysis. This is because, the higher elevation of the 
southern field exceeded the minimum requirements for ContextCapture software, which disrupted the computation process. However, the nadir flights at $125 \mathrm{ft}$ yielded the most consistent results, with the estimated volume of Pyramid A ranging between 14. 05cu.yd to 14. 42cu.yd in value ranging from $0.35 \%$ to $3.03 \%$ from actual volume. Pyramid B yielded values of between $4.94 \mathrm{cu}$.yd to $5.15 \mathrm{cu}$.yd. These volumes range from $1.22 \%$ to $3.01 \%$ of the actual volume. The values generated by images from $350 \mathrm{ft}$ had higher inaccuracies. The volumes computed for Pyramid A and B ranged from 5.22\% to $21.87 \%$ and $5.84 \%$ to $16.91 \%$ from actuals respectively. Table 2 provides a summary of the results of the experiment in cubic yards.

\section{Nadir flights with Oblique images}

In addition to the flights described in table 1, orbital way-point assisted flights were also flown capturing oblique images. These orbital flights were images captured at an angle of $\geq 45^{\circ}$ to the base center of each pyramid at the northern and southern sections of the field. These images were then labelled Oblique North and South and tested with the nadir flights. When these oblique images were added to the models the results did not improve significantly. Most of the oblique images from the northern and southern sections of the field were rejected by the software, and hence were not very reliable in supplying a detailed analysis of the models. However, the researchers were able to get a reading on the volume of Pyramid A at $40 \mathrm{ft}$ when the oblique images were added, as compared to just its nadir flight volume. The flights at $125 \mathrm{ft}$ were most consistent with yielding results, while flight at $350 \mathrm{ft}$ did not yield any results, as the software failed to estimate the block position between both sets of images.

The estimated volumes of each Pyramid at $125 \mathrm{ft}$ were compiled and compared to the volumes of the corresponding nadir flight values. The best results were provided at $125 \mathrm{ft}$, where a negligible change in values was observed with the addition of oblique images. Overall, an approximate $1 \%$ change in value was observed across each iteration with the addition of oblique images to the models. The resulting differences are shown in table 3 below.

Table 2

Volume calculation results from SfM software

\begin{tabular}{cccccccc}
\hline \multicolumn{3}{c}{ Pyramid A } & \multicolumn{6}{c}{ Pyramid B } \\
\hline Flight & $\begin{array}{c}\text { Computed } \\
\text { Vol. } \\
(\mathbf{c y})\end{array}$ & $\begin{array}{c}\text { Actual } \\
\text { Vol. } \\
\text { (cy) }\end{array}$ & $\begin{array}{c}\text { \% } \\
\text { Diff. }\end{array}$ & Flight & $\begin{array}{c}\text { Computed } \\
\text { Vol. } \\
\text { (cy) }\end{array}$ & $\begin{array}{c}\text { Actual } \\
\text { Vol } \\
\text { (cy) }\end{array}$ & $\begin{array}{c}\text { \% } \\
\text { Diff. }\end{array}$ \\
\hline $40 \mathrm{~A}$ & 0.00 & 14.00 & $\mathrm{n} / \mathrm{a}$ & $\mathbf{4 0 A}$ & 0.00 & 5.00 & $\mathrm{n} / \mathrm{a}$ \\
$40 \mathrm{~B}$ & 0.00 & 14.00 & $\mathrm{n} / \mathrm{a}$ & $\mathbf{4 0 B}$ & 0.00 & 5.00 & $\mathrm{n} / \mathrm{a}$ \\
$40 \mathrm{C}$ & 0.00 & 14.00 & $\mathrm{n} / \mathrm{a}$ & $\mathbf{4 0 C}$ & 0.00 & 5.00 & $\mathrm{n} / \mathrm{a}$ \\
$125 \mathrm{~A}$ & 14.42 & 14.00 & $3.03 \%$ & $\mathbf{1 2 5 A}$ & 5.15 & 5.00 & $3.01 \%$ \\
$125 \mathrm{~B}$ & 14.20 & 14.00 & $1.42 \%$ & $\mathbf{1 2 5 B}$ & 4.94 & 5.00 & $1.22 \%$ \\
$125 \mathrm{C}$ & 14.05 & 14.00 & $0.35 \%$ & $\mathbf{1 2 5 C}$ & 5.12 & 5.00 & $2.48 \%$ \\
$350 \mathrm{~A}$ & 17.06 & 14.00 & $21.87 \%$ & $\mathbf{3 5 0 A}$ & 5.85 & 5.00 & $16.91 \%$ \\
$350 \mathrm{~B}$ & 15.96 & 14.00 & $14.01 \%$ & $\mathbf{3 5 0 B}$ & 5.29 & 5.00 & $5.84 \%$ \\
$350 \mathrm{C}$ & 14.73 & 14.00 & $5.22 \%$ & $\mathbf{3 5 0 C}$ & 5.57 & 5.00 & $11.36 \%$ \\
\hline
\end{tabular}


Table 3

Comparison between estimated and measured volume values

\begin{tabular}{cccccccc}
\hline \multicolumn{3}{c}{ Pyramid A } & \multicolumn{5}{c}{ Pyramid B } \\
\hline $\begin{array}{c}\text { Computed } \\
\text { Vol. } \\
\text { Flights }\end{array}$ & $\begin{array}{c}\text { Actual } \\
\text { Vol. } \\
(\mathbf{c y})\end{array}$ & $\begin{array}{c}\text { \% } \\
\text { Diff. }\end{array}$ & Flights & $\begin{array}{c}\text { Computed } \\
\text { Vol. } \\
\text { (cy) }\end{array}$ & $\begin{array}{c}\text { Actual } \\
\text { Vol. } \\
\text { (cy) }\end{array}$ & $\begin{array}{c}\text { \% } \\
\text { Diff. }\end{array}$ \\
\hline $125 \mathrm{~A}$ & 14.42 & 14.00 & $3.03 \%$ & $125 \mathrm{~A}$ & 5.15 & 5.00 & $3.01 \%$ \\
$125 \mathrm{~B}$ & 14.20 & 14.00 & $1.42 \%$ & $125 \mathrm{~B}$ & 4.94 & 5.00 & $1.22 \%$ \\
$125 \mathrm{C}$ & 14.05 & 14.00 & $0.35 \%$ & $125 \mathrm{C}$ & 5.12 & 5.00 & $2.48 \%$ \\
$125 \mathrm{~A}+$ & & & & $125 \mathrm{~A}+$ & & & \\
Obliques & 14.32 & 14.00 & $2.27 \%$ & Obliques & 5.03 & 5.00 & $0.69 \%$ \\
$125 \mathrm{~B}+$ & & & & $125 \mathrm{~A}+$ & & & \\
Obliques & 14.31 & 14.00 & $2.22 \%$ & Obliques & 5.11 & 5.00 & $2.19 \%$ \\
$125 \mathrm{C}+$ & & & & $125 \mathrm{C}+$ & & & \\
Obliques & 14.07 & 14.00 & $0.47 \%$ & Obliques & 5.15 & 5.00 & $3.04 \%$ \\
\hline
\end{tabular}

\section{Oblique flights only}

As discussed previously, orbital images (Oblique North and Oblique South) were added to the nadir photo data set to improve model accuracy. These orbitals were extracted and processed independently from the nadir flights to test their accuracy against the actual values. Supplemental transition images were also captured on the north field at $35 \mathrm{ft}$ and $50 \mathrm{ft}$ to the base center of Pyramid A to compare values against the Oblique North and South images. These images were also processed separately from the nadir flights, and the values were compared to the actual values. Finally, all the orbitals and transitional images were combined into one model and analyzed. All images were accepted for use by the software for georeferencing. The results of the oblique models are detailed in table 4:

Table 4

Volume calculations using oblique imagery and

\begin{tabular}{|c|c|c|c|c|c|c|c|}
\hline \multicolumn{4}{|c|}{ Pyramid A } & \multicolumn{4}{|c|}{ Pyramid B } \\
\hline Flight & $\begin{array}{c}\text { Computed } \\
\text { Vol. } \\
\text { (cy) } \\
\end{array}$ & $\begin{array}{l}\text { Actual } \\
\text { Vol. } \\
\text { (cy) }\end{array}$ & $\begin{array}{c}\% \\
\text { Diff. }\end{array}$ & Flight & $\begin{array}{c}\text { Computed } \\
\text { Vol. } \\
\text { (cy) } \\
\end{array}$ & $\begin{array}{l}\text { Actual } \\
\text { Vol. } \\
\text { (cy) }\end{array}$ & $\begin{array}{c}\% \\
\text { Diff. }\end{array}$ \\
\hline $\begin{array}{l}\text { Oblique } \\
35+50\end{array}$ & 14.44 & 14.00 & $3.12 \%$ & $\begin{array}{l}\text { Oblique } \\
35+50\end{array}$ & N/A & 5.00 & $\mathrm{n} / \mathrm{a}$ \\
\hline $\begin{array}{c}\mathrm{N} \text { and } \mathrm{S} \\
\text { All }\end{array}$ & 14.21 & 14.00 & $1.52 \%$ & $\begin{array}{l}\text { Oblique } \\
\mathrm{N} \text { and } \mathrm{S} \\
\text { All }\end{array}$ & 4.59 & 5.00 & $8.23 \%$ \\
\hline Obliques & 14.06 & 14.00 & $0.44 \%$ & Obliques & 5.06 & 5.00 & $1.28 \%$ \\
\hline
\end{tabular}

The values of the oblique images were shown to be very close in value to the measured volumes of both Pyramid A and B. Pyramid A recorded a range in values between $14.06 \mathrm{cu}$. yd to $14.44 \mathrm{cu}$. yd. 
Pyramid B was not included in the values for the oblique flights at 35 degrees and 50 degrees as these images were concentrated only at the north field. However, Pyramid B showed similar values for the other oblique flights, with values of $4.59 \mathrm{cu}$.yd to $5.06 \mathrm{cu}$.yd. Overall, the combined oblique and transitional images proved to be the closest in value to the actuals. Pyramid A and B yielded values of $14.06 \mathrm{cu}$. yd and $5.06 \mathrm{cu}$. yd respectively, which translated into a range of $0.44 \%$ to $1.28 \%$ error recorded by the software.

\section{Conclusions and Future Study}

This experiment illustrates the cost effectiveness of aerial surveys when compared with traditional survey methods. It also contrasts the accuracy of stockpile measurements given conditions controllable by the drone operator. Overall, the flights at $125 \mathrm{ft}(125 \mathrm{~A}, 125 \mathrm{~B}$, and $125 \mathrm{C})$ yielded the closest results to the actual volumes of both Pyramid A and Pyramid B. Values yielded a minimum of $0.35 \%$ to a maximum of $3.03 \%$ difference when compared to the measured volumes. Flights at $350 \mathrm{ft}$ yielded results up to $22 \%$ off the actual volume of the stockpiles. As such, when measuring the volumes of stockpiles and other materials, it would be best for a contractor to maintain a lower altitude with standard overlap for best results. The addition of oblique images did not yield significantly better results for the volume calculation. This may have been due to the angle of the images exceeding the minimum required specifications for measure (focal length and altitude of flight). Testing of the oblique images only yielded a difference of up to $8 \%$ from the measured volumes of stockpile. Future study of these models could include the addition of tie points and extra transitional images to improve the accuracy. Also, a site with a less steep change in elevation will greatly aid in the capturing of images at lower altitudes which will improve the modelling process.

In conclusion, although conducting volumetric surveys with drones may seem to be less timeconsuming than traditional methods, the contractor would do well to set up enough GCPs and CPs to ensure the geo-positioning is effective enough for scaled measurements. Doing this will facilitate the mapping and computing of stockpile volumes. Also, properly transitioned oblique orbits are essential for the robustness of the models to improve the texture and accuracy of the models. An added advantage of the aerial volumetric survey is the ability to conduct flights over dangerous terrain in half the time it would usually take with other traditional methods.

\section{References}

AASHTO (2018). "Survey finds a growing number of State DOTS are deploying drones to improve safety and collect data faster and better-saving time and money." Transportation TV Special Report. [WWW document]. Drones'18cc.pdf

Aguera-Vega, F., Carvajal-Ramirez, F., \& Martinez-Carricondo, P. (2016). “Assessment of photogrammetric mapping accuracy based on variation ground control points number using unmanned aerial vehicle.” Measurement, 98, 221-227. ASCE Journal of Survey Engineering, Vol. 143. DOI: 10.1061/(ASCE)SU.1943-5428.0000206.

Bentley Reality Modeling Drone Capture Guide (2018). Bentley Systems, Acute3D, France. [WWW document]. Drone_capture_guide_EN.pdf

Draeyer, B., \& Strecha, C. (2014). "White paper: How accurate are UAV surveying methods?" [WWW document]. Pix4D White Paper. 
DJI Phantom 4 Pro V2.0 (2019). Website. Retrieved from url: https://www.dji.com/phantom-4-pro$\mathrm{v} 2$ ? site $=$ brandsite \&from=insite_search

El Meouche, R., Hijazi, I., Poncet, P. A., Abunemeh, M., \& Rezoug, M. (2016). “UAV photogrammetry implementation to enhance land surveying, comparisons and possibilities." International Archives of the Photogrammetry, Remote Sensing \& Spatial Information Sciences, Vol. 42 .

Ellenberg, A. Kontsos, A. Moon, F. \& Bartoli, I. (2016). "Bridge deck delamination identification from unmanned aerial vehicle infrared imagery." Automation in Construction. Vol. 72, Pages 155165 .

FAA, 2016. "Summary of Small Unmanned Aircraft rule (Part 107)". Federal Aviation Administration, Washington, DC. [WWW document]. URL https://www.faa.gov/uas/media/Part_107_Summary.pdf

Hugenholtz, C. H., Walker, J., Brown, O., \& Myshak, S. (2014). "Earthwork Volumetrics with an Unmanned Aerial Vehicle and softcopy photogrammetry". Journal of Surveying Engineering, Vol. 14.

Kim, H., Son, H., \& Kim, C. (2019). "Digital Terrain Modeling Using AKAZE Features Derived from UAV-Acquired, Nadir and Oblique Images." 36th International Symposium on Automation and Robotics in Construction (ISARC 2019).

Pix4D, 2018a. "Difference between a ground control point and a checkpoint." Website. Retrieved from url: https://support.pix4d.com/hc/en-us/articles/115000140963-Difference-between-a-groundcontrol-point-and-a-checkpoint

Pix4D, 2018b. "How to import and mark Manual Tie Points." Website. Retrieved from url: https://support.pix4d.com/hc/en-us/articles/360000395103-How-to-import-and-mark-Manual-TiePoints-MTPs-

UAV Coach, 2019. "Top 100 Drone Companies to Watch in 2019”. Website. Retrieved from url: https://uavcoach.com/drone-companies/

Vincenzi, D. A., Terwilliger, B. A., Ison, D. C. (2015). "Unmanned Aerial System (UAS) HumanMachine Interfaces: New Paradigms in Command and Control.” Procedia Manufacturing, Vol 3, 2015. Pages 920-927. 